\title{
Angiographic, hemodynamic, and histological changes in an animal model of brain arteriovenous malformations treated with Gamma Knife radiosurgery
}

\author{
Saleh R. Kashba, MB, BCh, ${ }^{1}$ Nirav J. Patel, MD, ${ }^{1}$ Michael Grace, BMedPhys, MACPSEM, ${ }^{2}$ \\ Vivienne S. Lee, BLabMed, ${ }^{1}$ Newsha Raoufi-Rad, BBiotech(Hons), ${ }^{1}$ \\ Jude V. Amal Raj, BSc, MBiotech, ${ }^{1}$ Thi Thuy Hong Duong, PhD, ${ }^{1}$ and \\ Marcus Stoodley, PhD, FRACS ${ }^{1}$
}

\begin{abstract}
${ }^{1}$ Australian School of Advanced Medicine, Macquarie University; and ${ }^{2}$ Genesis Cancer Care, Macquarie University Hospital, Sydney, New South Wales, Australia
\end{abstract}

\begin{abstract}
OBJECT Brain arteriovenous malformations (AVMs) are a major cause of stroke. Many AVMs are effectively obliterated by stereotactic radiosurgery, but such treatment for lesions larger than $3 \mathrm{~cm}$ is not as effective. Understanding the responses to radiosurgery may lead to new biological enhancements to this treatment modality. The aim of the present study was to investigate the hemodynamic, morphological, and histological effects of Gamma Knife surgery (GKS) in an animal model of brain AVM.
\end{abstract}

METHODS An arteriovenous fistula was created by anastomosing the left external jugular vein to the side of the common carotid artery in 64 male Sprague-Dawley rats (weight $345 \pm 8.8 \mathrm{~g}$ ). Six weeks after AVM creation, 32 rats were treated with a single dose of GKS (20 Gy); 32 animals received sham radiation. Eight irradiated and 8 control animals were studied at each specified time point $(1,3,6$, and 12 weeks) for hemodynamic, morphological, and histological characterization.

RESULTS Two AVMs showed partial angiographic obliteration at 6 weeks. Angiography revealed complete obliteration in 3 irradiated rats at 12 weeks. Blood flow in the ipsilateral proximal carotid artery $(p<0.001)$ and arterialized jugular vein $(p<0.05)$ was significantly lower in the irradiated group than in the control group. The arterialized vein's external diameter was significantly smaller in GKS-treated animals at $6(p<0.05)$ and $12(p<0.001)$ weeks. Histological changes included subendothelial cellular proliferation and luminal narrowing in GKS-treated animals. Neither luminal obliteration nor thrombus formation was identified at any of the time points in either irradiated or nonirradiated animals.

CONCLUSIONS GKS produced morphological, angiographic, and histological changes in the model of AVM as early as 6 weeks after treatment. These results support the use of this model for studying methods to enhance radiation response in AVMs.

http://thejns.org/doi/abs/10.3171/2014.10.JNS1435

KEY WORDS arteriovenous malformation; Gamma Knife; animal model; stereotactic radiosurgery

B RAIN arteriovenous malformations (AVMs) are a major cause of stroke in children and young adults. Surgical excision of an AVM offers immediate protection from hemorrhage and is suitable for small and superficial lesions. Approximately $70 \%$ of small AVMs $(<3$ $\mathrm{cm}$ in diameter) are completely obliterated by $2-3$ years after Gamma Knife surgery (GKS)., ${ }^{1,323}$ However, patients treated with GKS remain at risk for suffering hemorrhage during the latent period before AVM occlusion. ${ }^{9,15}$ A large number of patients who have large and/or deeply located AVMs cannot be treated using current methods. ${ }^{9}$ An improved method of treating AVMs is required for these patients.

One potential new treatment is to induce thrombosis in

ABBREVIATIONS AVM = arteriovenous malformation; GKS = Gamma Knife surgery; LCCA = left common carotid artery; LEJV = left external jugular vein; LINAC = linear accelerator.

SUBMITTED February 8, 2014. ACCEPTED October 16, 2014.

INCLUDE WHEN CITING Published online April 17, 2015; DOI: 10.3171/2014.10.JNS1435.

DISCLOSURE This study was supported by grants from the Australian National Health and Medical Research Council, the Royal Australasian College of Surgeons, and Elekta Australia \& New Zealand. 
the AVM vessels using molecular techniques that target endothelial changes induced by radiosurgery. Development of such a treatment requires an animal model that mimics the characteristics of human AVMs. We have developed an animal model that shares hemodynamic, structural, and molecular characteristics with human AVMs. ${ }^{6,20}$ These characteristics make this model suitable for studying the early molecular responses of the AVM endothelial phenotype to radiation. However, before studying the molecular responses, one must understand the morphological, hemodynamic, and histological changes that occur after radiation treatment. We have previously studied the effects of linear accelerator (LINAC) radiosurgery, ${ }^{16,19}$ but the effects of GKS in this model have not been studied. The aim of the present study was to investigate the morphological, hemodynamic, and histological changes in the animal model of AVM treated with GKS.

\section{Methods}

\section{Surgical Fistula Formation}

All studies were approved by the Animal Care and Ethics Committee at Macquarie University. Animal experimentation was performed in accordance with this committee's guidelines and the Australian Code of Practice for the Care and Use of Animals for Scientific Purposes.

The AVM model was created in 64 male Sprague-Dawley rats (weight $345 \pm 8.8 \mathrm{~g}$ ). The model creation has been described in detail previously. ${ }^{24}$ Briefly, rats were anesthetized by isoflurane inhalation. The left common carotid artery (LCCA) and left external jugular vein (LEJV) were exposed under an operating microscope (Carl Zeiss). The LEJV was ligated at its junction with the subclavian vein with 10-0 monofilament nylon suture (Ethicon). Mobilization of the LEJV was achieved by coagulation of no more than 2 side branches, and care was taken not to injure the LEJV. The end of the LEJV was anastomosed to the side of the LCCA with continuous 10-0 monofilament nylon suture (Ethicon). A monofilament nylon suture (4-0) (Ethicon) was used to close the wound in a single layer.

\section{Gamma Knife Surgery}

Thirty-two male rats were treated using a single-fraction stereotactic radiosurgical dose administered using the Leksell Gamma Knife Perfexion (Elekta) 6 weeks after AVM creation. The animals were sedated using ketamine $(75 \mathrm{mg} / \mathrm{kg})$ and medetomidine $(0.5 \mathrm{mg} / \mathrm{kg})$. Adequate anesthesia was determined by the hindlimb pinch test. Animals were then placed and immobilized in the supine position in a specially designed frame (Genesis Cancer Care, Australia). Axial full-body CT scanning with 3D reconstruction was performed for AVM localization (Fig. 1). The skin surface was defined, the nidus was identified, and an appropriately small- or fine-dose grid was placed over the area of the nidus for accurate dosimetry. The model AVM nidus was then treated stereotactically with a 20 -Gy dose of radiation to the $50 \%$ isodose line in a single fraction, and particular care was taken to ensure the 10-Gy isodose line did not touch or traverse the esophagus or trachea. Tight shaping of the radiation dose to the nidus or anastomosis is deliberately avoided because an overly tight radiation dose to the nidus will reduce the dose homogeneity in the higher-dose region. An unblocked 8-mm shot (placed in this position to restrict the 10-Gy isodose from touching the esophagus) has been found to be the most reproducible while also providing the highest dose homogeneity and uniformity in the high-dose region. AVMs were created for the nonirradiated 32 control animals, but they did not receive any radiation.

\section{Angiography}

Eight control and 8 GKS-treated animals were studied at each time point (1, 3, 6, and 12 weeks). Angiography was performed immediately prior to sacrifice. Animals were placed under general anesthesia using 2\%-3\% isoflurane in oxygen. Under the operating microscope, the left femoral artery was exposed. A 0.8 -mm Progreat microcatheter (Terumo) was advanced through a small arteriotomy under fluoroscopic C-arm (GE Healthcare) control up to the LCCA. An arterial-phase anteroposterior angiogram was obtained after manual injection of $1 \mathrm{ml} / \mathrm{kg}$ contrast medium (meglumine iotalamate, $60 \% \mathrm{w} / \mathrm{v}$, Covidien Pty Ltd) over 3 seconds. The catheter was withdrawn, and the femoral artery was ligated after completion of angiography. Angiographic measurements were made against a standard control object of known diameter (a coin).

\section{Arterialized Vein Diameter}

Each fistula was exposed after completion of angiography. A 4-0 silk suture was applied around the proximal segment of the arterialized external jugular vein to measure the circumference and calculate the diameter (circumference/ $\pi$ ).

\section{Hemodynamic Studies}

Blood flow measurements were obtained from the
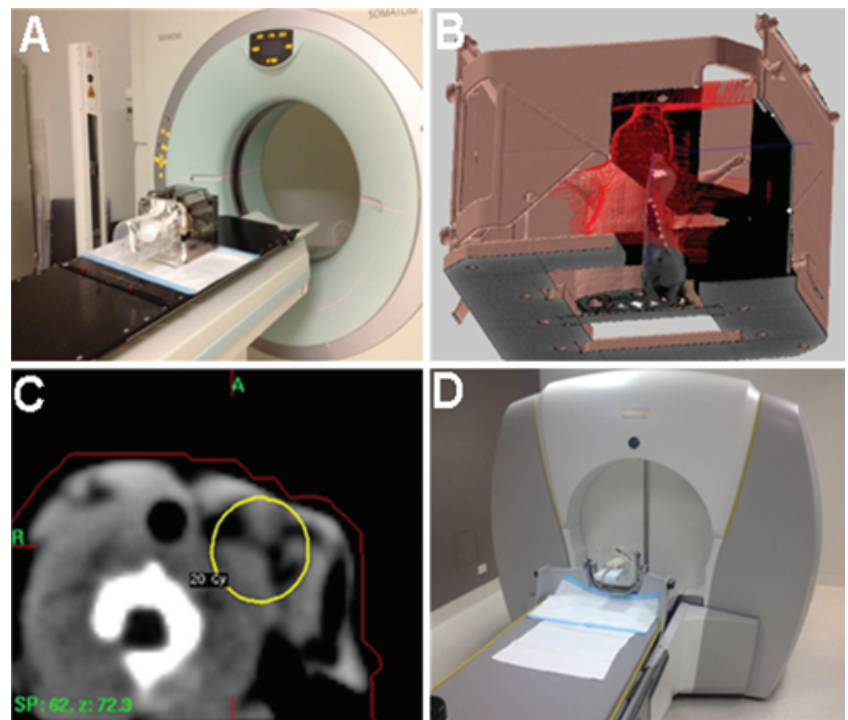

FIG. 1. GKS planning and treatment. A: Each rat is placed in a frame, and a whole-body CT scan is obtained. B: 3D reconstruction demonstrating the position of the animal in the frame. C: Axial CT scan of the rat showing the margin dose to the AVM nidus. D: Anesthetized animal on the frame about to enter the Gamma Knife unit for treatment. 
proximal LCCA and the LEJV using a Doppler flowmeter. Perivascular flow probes (Transonic Systems) were selected to match the size of the vessel in which flow was being measured. Probes were connected to a transit time perivascular flowmeter (TS420, Transonic Systems). The average during a 10-minute recording was used to determine the blood flow. Measurements of blood pressure were performed simultaneously in the left femoral artery (for mean systemic arterial pressure). A $0.58-\mathrm{mm}$ polyethylene catheter was inserted into the vessel, and pressure was measured with a blood pressure transducer (Edwards Lifesciences). Flow and pressure data were acquired via a data acquisition system for off-line analysis (CED Limited).

\section{Histological Study}

Animals were killed by transcardial perfusion of phosphate-buffered saline with pulsatile flow at $28 \mathrm{ml} / \mathrm{minute}$. Different parts of the fistula were collected and embedded in freezing medium (ProSciTech) and were frozen in liquid nitrogen. Vascular tissues collected as controls included the contralateral jugular vein and carotid artery and the inferior vena cava. The tissues were sectioned with a thickness of $10 \mu \mathrm{m}$ and were stained with $\mathrm{H} \& \mathrm{E}$ or Shikata orcein, or they were immunostained for von Willebrand factor for endothelial staining.

\section{Light Microscopy and Data and Statistical Analysis}

Microscopy was performed using a Zeiss microscope (Carl Zeiss). Digital images were captured under fixed parameters with an AxioCam HRc digital camera (Carl Zeiss). ImageJ open-source software was used to measure the diameters of the proximal and distal parts of the LEJV and the proximal part of the LCCA. Blood flow analysis was performed using Spike (CED Limited). All data are presented as mean \pm SD. Statistical analysis was performed by 2 -way ANOVA. A p value $<0.05$ was considered statistically significant.

\section{Results}

Angiography

The fistula was patent in all control animals. In the
GKS-treated group, 2 animals had partial obliteration at 6 weeks, and 3 animals had complete obliteration at 12 weeks (Fig. 2). At the 1-week and 3-week time points, there were no significant differences between the control and treated groups in proximal and distal LEJV and LCCA diameters (Fig. 3). At 12 weeks there were significant differences in proximal and distal LEJV diameters between the treatment group and the control group $(\mathrm{p}<0.05)$.

\section{Hemodynamic Study}

In the control group, blood flow increased with time. However, in the GKS-treated group the LCCA flow increased until 3 weeks and then decreased or stabilized (Fig. 4). The treated group had a lower flow when assessed across all time points using ANOVA ( $\mathrm{p}<0.001)$. At 12 weeks, there was a significantly lower LCCA flow rate in the treated group compared with that in the control group $(p<0.001)$. Using ANOVA, we found a significant difference $(p<0.05)$ in flow in the LEJV in animals in the treated group compared with that in the control group. However, individual time-point comparisons between the 2 groups did not show any statistically significant differences (Fig. 4).

\section{Histological Changes}

No histological changes were identified in the control vascular tissue (contralateral jugular vein and carotid artery and inferior vena cava). Irradiated AVMs showed no significant changes at 1 or 3 days, or at 1 or 3 weeks. Concentric subendothelial cell growth was identified in some irradiated AVMs at 6 weeks and 12 weeks, along with vessel wall thickening and luminal narrowing. Neither luminal obliteration nor thrombus formation was identified at any of the time points (Fig. 5). The elastic lamina had multiple layers in both groups, and no changes occurred in either group over time (Fig. 6). The endothelial lining remained intact in both groups at all time points (Fig. 7).

\section{Arterialized Vein Diameter and Morphology}

The LEJV external diameter was significantly smaller in the treated animals than the control animals at 6 weeks $(\mathrm{p}<0.05)$ and 12 weeks $(\mathrm{p}<0.001)$ (Fig. 8). Whitish fi-
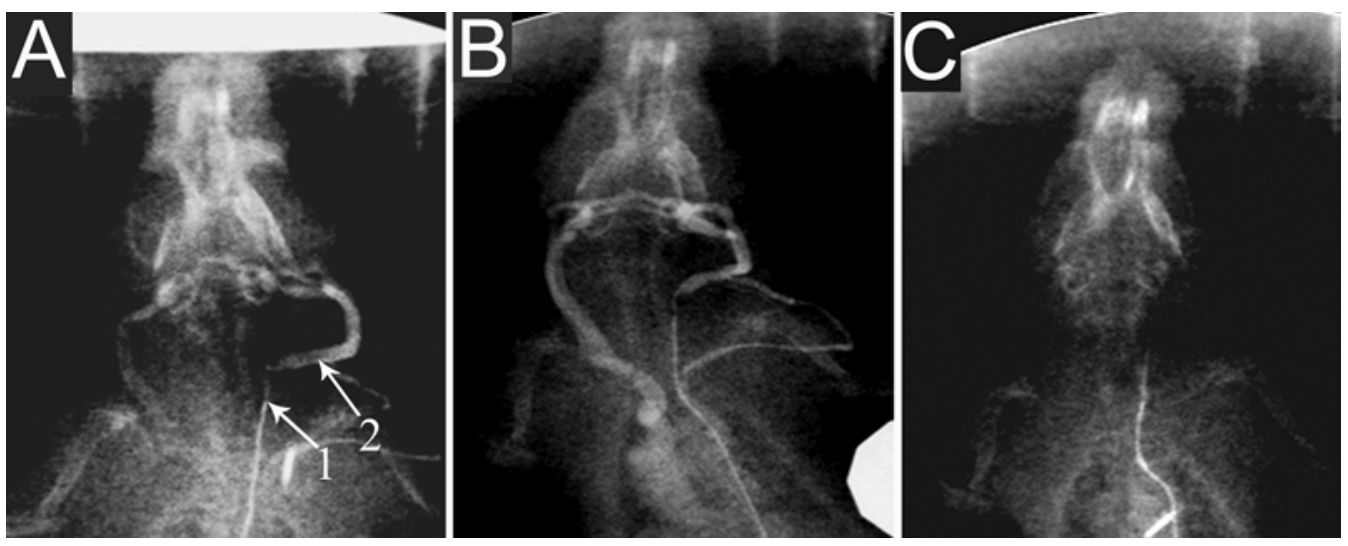

FIG. 2. Angiograms of the AVM model at 12 weeks. A: Control animal. The carotid artery (1) and fistula are patent, and the jugular vein (2) is enlarged. B: Animal treated with GKS. The fistula is patent, but the jugular vein is narrowed. C: Animal treated with GKS. The fistula is occluded. 

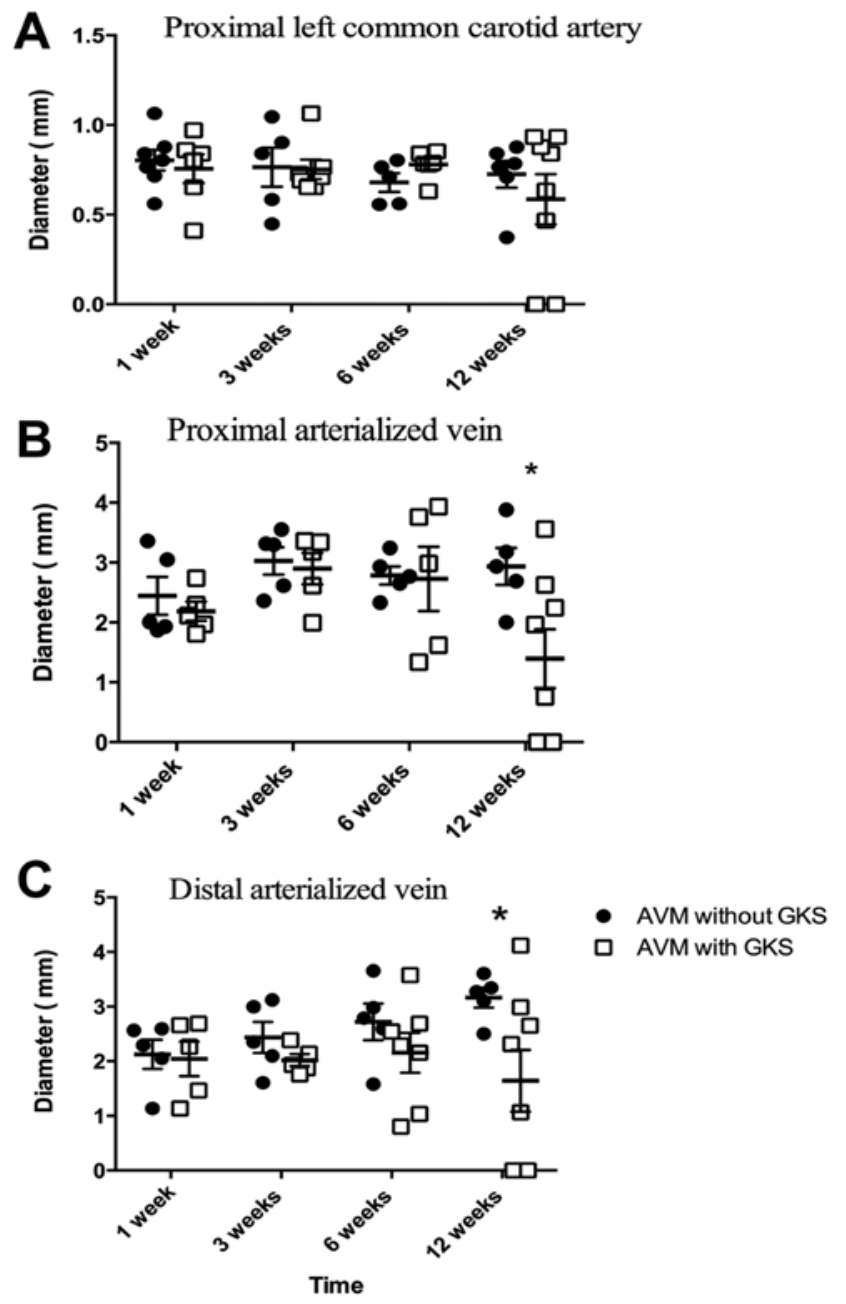

FIG. 3. Angiographically measured vessel diameters in control and radiated animals. A: Proximal LCCA internal diameter $(F[7,42]=2.024$; $p>0.05)$. B: Proximal arterialized LEJV internal diameter $(F[7,35]=$ 3.396; $p<0.05)$. C: Distal arterialized LEJV internal diameter ( $F[7,37]$ $=4.068 ; p<0.05)$.

brinous material was seen in the wall of the LEJV in the majority of treated animals; this was not seen in the control animals. Perivascular fibrous adhesions were seen in most of the treated animals at 6 and 12 weeks. Hematoma was also seen in tissue around treated AVMs at 1 week and 3 weeks.

\section{Discussion}

Animal models of human AVMs are needed for a variety of reasons. Biological models can contribute to the understanding of AVM pathogenesis and rupture. ${ }^{20,24}$ They could also be used to study the effects of radiosurgery because this treatment method's mechanism of action is not well understood. ${ }^{12}$ Models in large animals can be used to develop endovascular treatments and to train operators in their use. Our need for an animal model is driven by our desire to develop a new molecular medicine-based treatment for AVMs, many of which cannot be treated safely or effectively with current methods., ${ }^{4,9,11}$ Although the model we have used here is not an intraparenchymal brain AVM,
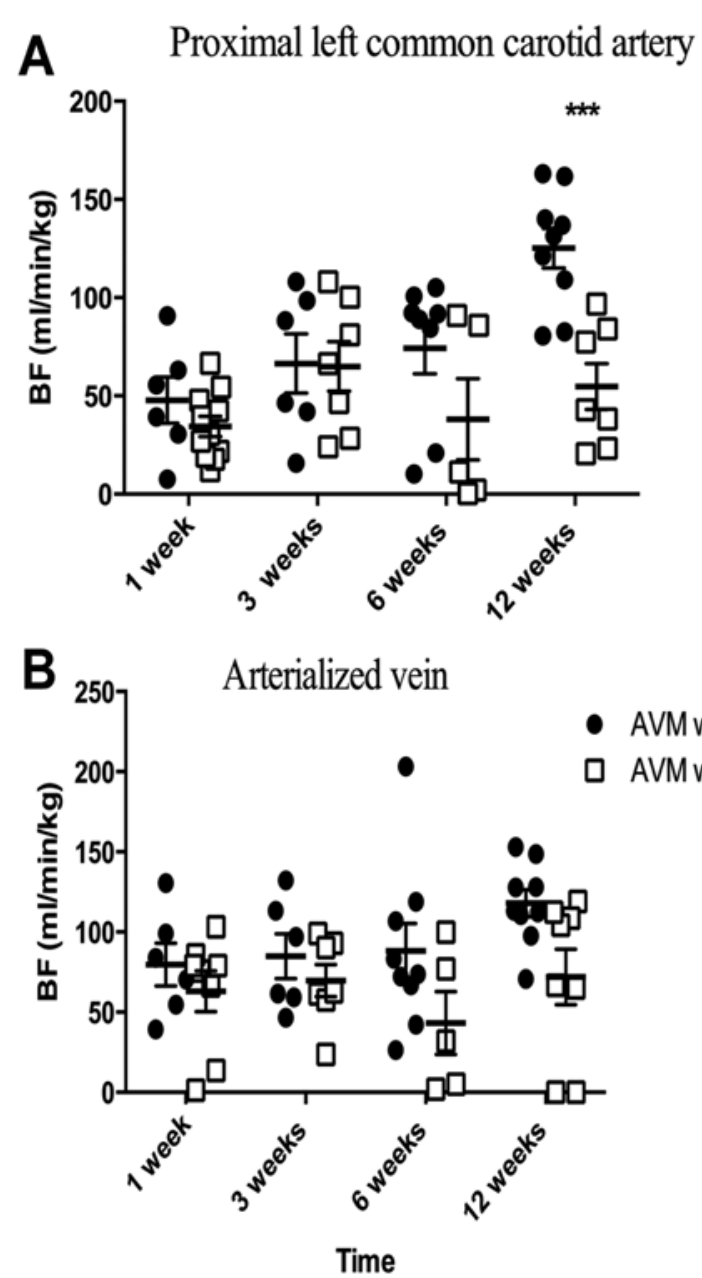

FIG. 4. Blood flow (BF) measured with Doppler flowmeter. A: Proximal LCCA flow. The flow was significantly lower in the treated group at 12 weeks $(F[7,51]=0.6998 ; p<0.001)$. B: Flow in the LEJV. The treated group had a lower flow than the control group $(F[7,50]=0.6113 ; p<$ 0.05).

it is the best model available for this study because its endothelial characteristics closely resemble those of human brain AVMs. ${ }^{6}$ Our long-term goal is to develop a molecular medicine-based treatment for AVMs by exploiting endothelial surface molecular changes induced by radiation; such molecular changes may help identify suitable targets for the delivery of biologically active agents such as prothrombotic molecules. Because radiation effects vary among endothelial phenotypes, it is necessary to use a model that replicates the endothelial phenotype of human AVMs. A model also must have a definable target volume that includes vessels with the AVM endothelial phenotype. The model we used here is the best available for these requirements. In addition, we focused on short-term molecular changes rather than the long-term processes of radiation-induced occlusion. Although this model may be suitable for the study of the occlusive effects of Gamma Knife radiation, that was not our focus. Accordingly, we have studied changes only out to 12 weeks after irradiation.

The overall goal of our research is to develop a new 

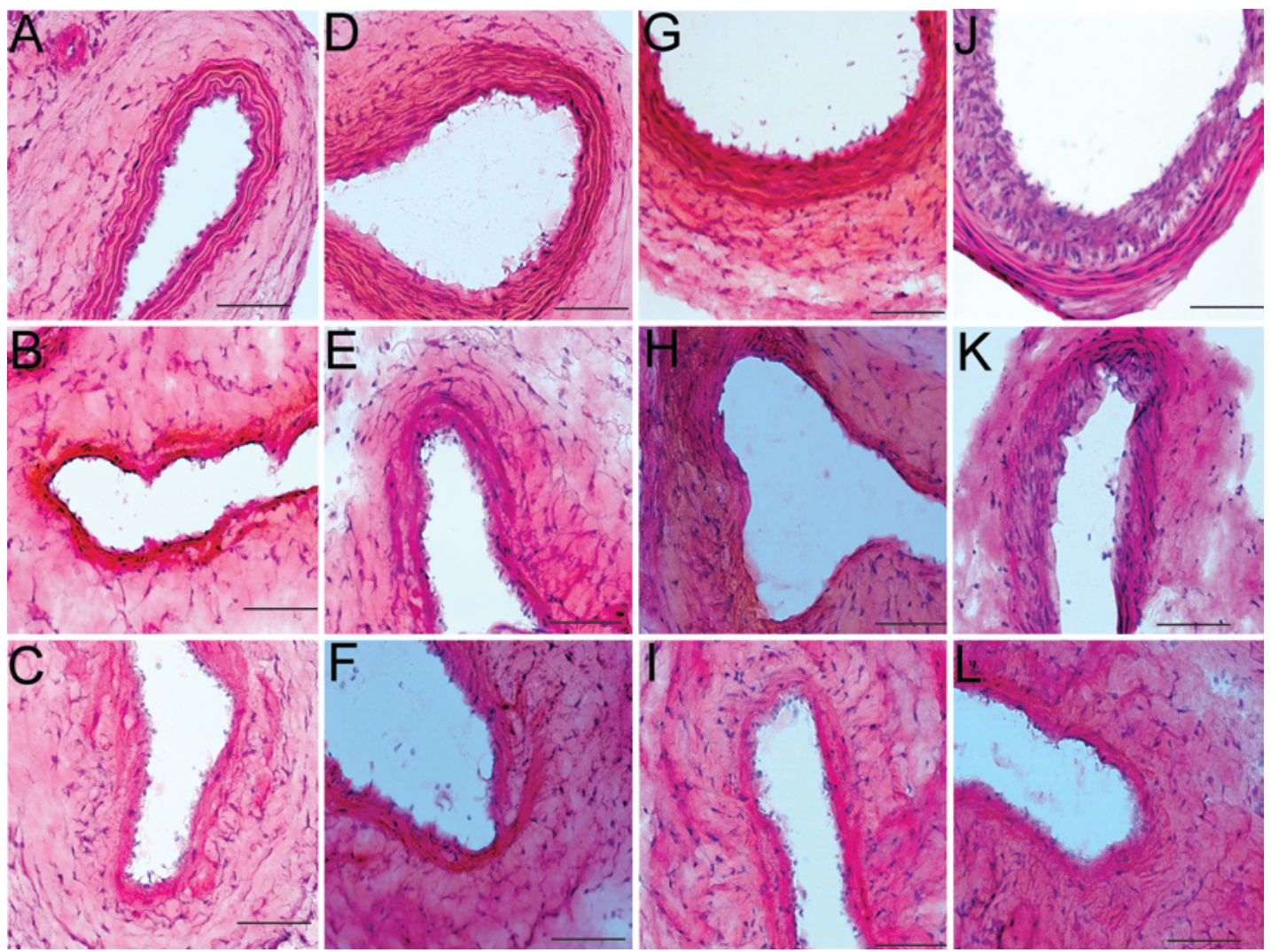

FIG. 5. Histological appearances in control $(\mathbf{A}-\mathbf{F})$ and irradiated $(\mathbf{G}-\mathbf{L})$ animals at 1 week (control $\mathbf{A}-\mathbf{C}$; irradiated $\mathbf{G}-\mathbf{I})$ and 12 weeks (control D-F; radiated J-L). Sections from the carotid artery are shown in the upper row $(A, D, G$, and J); sections from the arterialized vein are shown in the center row $(\mathrm{B}, \mathrm{E}, \mathrm{H}$, and $\mathrm{K})$; and sections of the nidus are shown in the lower row $(\mathrm{C}, \mathrm{F}, \mathrm{I}$, and $\mathrm{L})$. $H \& E$, original magnification $\times 20$, bar $=200 \mu \mathrm{m}$.

treatment of AVMs that involves rapid stimulation of thrombosis within the abnormal vessels. We propose that prothrombotic agents could be administered systemically but could be targeted to accumulate selectively within AVM vessels. Such targeting could involve attaching the prothrombotic agents to antibodies (or other specific binding molecules) that target endothelial surface molecules that are expressed in AVM vessels but not in normal vessels. Because it is a powerful inducer of endothelial molecular change $\mathrm{e}^{8,19}$ and provides precise spatial localization, radiosurgery could be used as a first step in this treatment to stimulate discriminating endothelial molecular changes within only AVM vessels. The successful development of this treatment will rely on an animal model that mimics
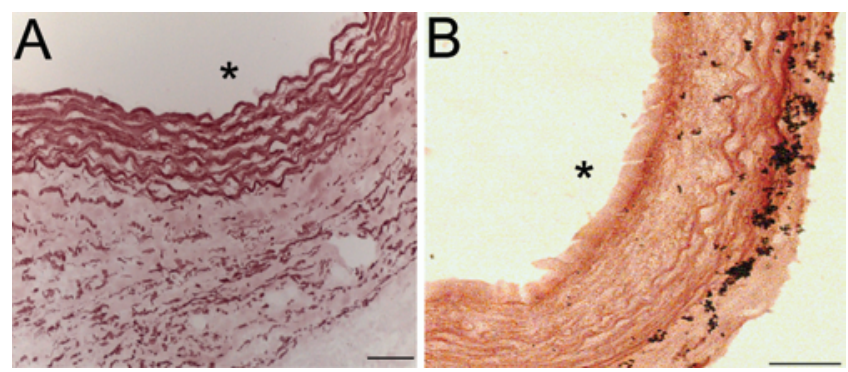

FIG. 6. Elastic lamina of the LCCA in a control animal (A) and GKStreated animal $(B)$ at 12 weeks. Asterisk shows the lumen. Shikata orcein stain, original magnification $\times 20$. the structural, hemodynamic, and molecular characteristics of human AVMs and that can be treated with radiosurgery. The endothelial phenotype varies widely depending on the organ of origin, and AVM endothelium also differs from normal cerebral endothelium because of its molecular characteristics. ${ }^{16,17}$ The response of the endothelium to radiation may vary with different phenotypes, so our research developing a molecular treatment that targets radiation-induced endothelial changes must be done using a model that mimics the human AVM phenotype and its response to radiation.

The animal model used here has been shown previously to mimic human AVMs in its structural, hemodynamic, angiographic, and molecular characteristics. ${ }^{20} \mathrm{We}$ also developed a method to treat the model AVM with LINAC radiosurgery and studied changes in adhesion molecules after irradiation..$^{19}$ The feasibility of the proposed thrombotic treatment was demonstrated using LINAC as the radiosurgery priming method. ${ }^{13,18}$ We now have access to GKS and can use 3D CT scanning to help plan GKS for each rat that is treated. We intend to continue this research using GKS rather than LINAC. However, the responses of the AVM model to GKS are not necessarily the same as the responses to LINAC, and a detailed study of responses to GKS was required before moving on to trials of new molecular treatments.

The change to GKS involved the development of a new frame and individual treatment planning based on 3D CT 

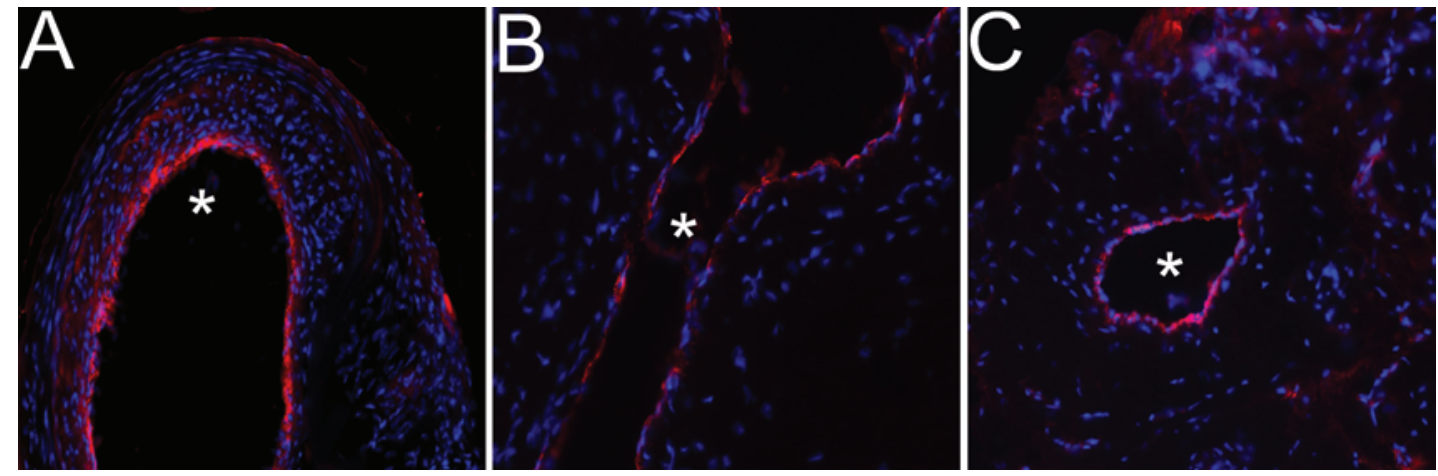

FIG. 7. Endothelial staining with von Willebrand factor (red) and 4',6-diamidino-2-phenylindole (DAPI) (blue) in a radiated animal at 12 weeks. A: LCCA. B: Arterialized LEJV. C: Nidus. Asterisk shows the lumen.

scans. The carotid artery and the arterialized jugular vein are readily identified on the noncontrast $\mathrm{CT}$ scans, and treatment planning is relatively straightforward (Fig. 1).

GKS resulted in angiographic, hemodynamic, and structural changes that were evident by 6 weeks after treatment. Angiography revealed narrowing or occlusion of the arterialized vein in animals at 6 and 12 weeks, although complete occlusion of the vein was not confirmed histologically. Following treatment, blood flow was lower at 6 and 12 weeks in the GKS-treated animals. The diameter of the arterialized vein was also smaller at the later time points. Vessel wall thickening, perivascular fibrosis, and subendothelial cellular proliferation were observed at 6 and 12 weeks.

Very few studies have examined radiosurgery in animal models of AVM. De Salles et al. used LINAC doses from 20 to 90 Gy to treat the rete mirabile in pigs. They found no angiographic changes 2 months after treatment, and substantial reductions in vascularity occurred only with
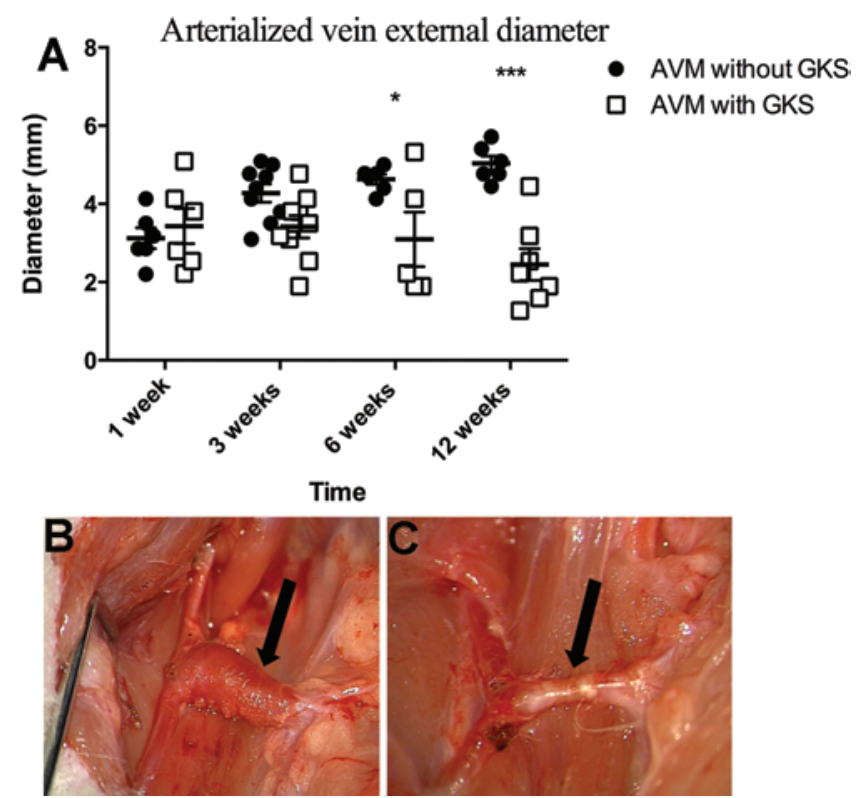

FIG. 8. A: External diameter of the arterialized LEJV. The vein diameter was significantly smaller in the treated group at $6(p<0.05)$ and $12(p<$ 0.001 ) weeks. B: Nonirradiated AVM model. C: Irradiated AVM showing narrowing of the external diameter of the jugular vein and whitish fibrinous material in the vessel wall. Arrows indicate the arterialized vein. doses of 50 Gy or more. ${ }^{2}$ They reported histological effects similar to those reported here, including intimal proliferation that correlated with the dose of radiation. Reports of the effects of radiosurgery on normal vessels include a study of GKS on cat basilar artery ${ }^{10}$ and on rat anterior cerebral artery. ${ }^{5}$ Findings included intimal proliferation but no vessel occlusion except in 1 rat anterior cerebral artery 20 months after treatment with $100 \mathrm{~Gy}$.

The morphological changes in our treated animals were similar to the reported effects of radiosurgery in human AVMs; the latter included perivascular fibrosis, subendothelial proliferation, and luminal narrowing. ${ }^{14,21,22} \mathrm{Al}-$ though it is not possible to perform a detailed study of the short-term effects of GKS in human AVMs, it seems reasonable to infer that the effects in our model simulate the processes that occur in human AVMs.

Development of a successful prothrombotic treatment strategy for AVMs will depend on identification of a highly discriminating endothelial surface marker in AVMs that have been treated with radiosurgery. We are currently using the model described here, including treatment with GKS, in a search for the most prospective targets.

\section{Conclusions}

The hemodynamic and structural responses of the animal model following GKS treatment are likely to be similar to the changes that occur in treated human AVMs. This model is suitable for further study to identify highly discriminating endothelial molecular changes after radiosurgery that could be used as targets for prothrombotic molecular treatments.

\section{References}

1. Coffey RJ, Nichols DA, Shaw EG: Stereotactic radiosurgical treatment of cerebral arteriovenous malformations. Mayo Clin Proc 70:214-222, 1995

2. De Salles AA, Solberg TD, Mischel P, Massoud TF, Plasencia A, Goetsch S, et al: Arteriovenous malformation animal model for radiosurgery: the rete mirabile. AJNR Am J Neuroradiol 17:1451-1458, 1996

3. Guo WY, Lindquist C, Karlsson B, Kihlström L, Steiner L: Gamma knife surgery of cerebral arteriovenous malformations: serial MR imaging studies after radiosurgery. Int J Radiat Oncol Biol Phys 25:315-323, 1993

4. Han PP, Ponce FA, Spetzler RF: Intention-to-treat analysis of Spetzler-Martin grades IV and V arteriovenous malforma- 
tions: natural history and treatment paradigm. J Neurosurg 98:3-7, 2003

5. Kamiryo T, Lopes MBS, Berr SS, Lee KS, Kassell NF, Steiner L: Occlusion of the anterior cerebral artery after Gamma Knife irradiation in a rat. Acta Neurochir (Wien) 138:983-991, 1996

6. Karunanyaka A, Tu J, Watling A, Storer KP, Windsor A, Stoodley MA: Endothelial molecular changes in a rodent model of arteriovenous malformation. J Neurosurg 109:1165-1172, 2008

7. Klopfenstein JD, Spetzler RF: Cerebral arteriovenous malformations: when is surgery indicated? Acta Neurochir (Wien) 147:693-695, 2005

8. Liu S, Sammons V, Fairhall J, Reddy R, Tu J, Duong TT, et al: Molecular responses of brain endothelial cells to radiation in a mouse model. J Clin Neurosci 19:1154-1158, 2012

9. Maruyama K, Kawahara N, Shin M, Tago M, Kishimoto J, Kurita $\mathrm{H}$, et al: The risk of hemorrhage after radiosurgery for cerebral arteriovenous malformations. N Engl J Med 352:146-153, 2005

10. Nilsson A, Wennerstrand J, Leksell D, Backlund EO: Stereotactic gamma irradiation of basilar artery in cat. Preliminary experiences. Acta Radiol Oncol Radiat Phys Biol 17:150160, 1978

11. Nozaki K, Hashimoto N, Miyamoto S, Kikuchi H: Resectability of Spetzler-Martin grade IV and V cerebral arteriovenous malformations. J Clin Neurosci 7 Suppl 1:78-81, 2000

12. O'Connor MM, Mayberg MR: Effects of radiation on cerebral vasculature: a review. Neurosurgery 46:138-151, 2000

13. Reddy R, Duong TT, Fairhall JM, Smee RI, Stoodley MA: Durable thrombosis in a rat model of arteriovenous malformation treated with radiosurgery and vascular targeting. J Neurosurg 120:113-119, 2014

14. Schneider BF, Eberhard DA, Steiner LE: Histopathology of arteriovenous malformations after gamma knife radiosurgery. J Neurosurg 87:352-357, 1997

15. Shin M, Kawahara N, Maruyama K, Tago M, Ueki K, Kirino $\mathrm{T}$ : Risk of hemorrhage from an arteriovenous malformation confirmed to have been obliterated on angiography after stereotactic radiosurgery. J Neurosurg 102:842-846, 2005

16. Storer KP, Tu J, Karunanayaka A, Morgan MK, Stoodley MA: Inflammatory molecule expression in cerebral arteriovenous malformations. J Clin Neurosci 15:179-184, 2008

17. Storer KP, Tu J, Karunanayaka A, Morgan MK, Stoodley MA: Thrombotic molecule expression in cerebral vascular malformations. J Clin Neurosci 14:975-980, 2007
18. Storer K, Tu J, Karunanayaka A, Smee R, Short R, Thorpe P, et al: Coadministration of low-dose lipopolysaccharide and soluble tissue factor induces thrombosis after radiosurgery in an animal arteriovenous malformation model. Neurosurgery 61:604-611, 2007

19. Storer KP, Tu J, Stoodley MA, Smee RI: Expression of endothelial adhesion molecules after radiosurgery in an animal model of arteriovenous malformation. Neurosurgery 67:976-983, 2010

20. Tu J, Karunanayaka A, Windsor A, Stoodley MA: Comparison of an animal model of arteriovenous malformation with human arteriovenous malformation. J Clin Neurosci 17:96-102, 2010

21. Tu J, Stoodley MA, Morgan MK, Storer KP: Responses of arteriovenous malformations to radiosurgery: ultrastructural changes. Neurosurgery 58:749-758, 2006

22. Tu J, Stoodley MA, Morgan MK, Storer KP, Smee R: Different responses of cavernous malformations and arteriovenous malformations to radiosurgery. J Clin Neurosci 16:945-949, 2009

23. Yamamoto M, Jimbo M, Kobayashi M, Toyoda C, Ide M, Tanaka N, et al: Long-term results of radiosurgery for arteriovenous malformation: neurodiagnostic imaging and histological studies of angiographically confirmed nidus obliteration. Surg Neurol 37:219-230, 1992

24. Yassari R, Sayama T, Jahromi BS, Aihara Y, Stoodley M, Macdonald RL: Angiographic, hemodynamic and histological characterization of an arteriovenous fistula in rats. Acta Neurochir (Wien) 146:495-504, 2004

\section{Author Contributions}

Conception and design: Stoodley, Kashba, Raoufi-Rad, Raj, Duong. Acquisition of data: Stoodley, Kashba, Patel, Grace, Lee, Raoufi-Rad, Raj. Analysis and interpretation of data: Stoodley, Kashba. Drafting the article: Stoodley, Kashba. Critically revising the article: Stoodley, Kashba. Reviewed submitted version of manuscript: all authors. Approved the final version of the manuscript on behalf of all authors: Stoodley. Statistical analysis: Kashba. Study supervision: Stoodley.

\section{Correspondence}

Marcus Stoodley, Neurosurgery Unit, Australian School of Advanced Medicine, Ste. 201, Level 2, 2 Technology Pl., Macquarie University, Sydney, NSW 2109, Australia. email: marcus. stoodley@mq.edu.au. 